\title{
Barium sulfate crystallization in non-aqueous solvent
}

\author{
Ryan Fillingham, Matthew Boon, Shaghraf Javaid, J. Alex Saunders, Franca Jones*
}

Received 00th January 20xx, Accepted 00th January 20xx

DOI: $10.1039 / \times 0 \times x 00000 x$

\begin{abstract}
Crystallisation is performed in a non-aqueous solvent, dimethylsulfoxide (DMSO), in order to determine what the role of water is on the crystallisation pathway. In both water and DMSO environments the particles do not appear to grow by ion addition but rather appear to grow through aggregation. The main difference in water is that the aggregation processes are not random and result in particle morphologies that bear relation to the single-crystal faces, suggesting an oriented attachment mechanism. In DMSO, the aggregation processes appear less oriented and while there is some lattice registry, lattice mismatch is also observed and the aggregate shape is spherical overall. This is confirmed in the $1200 \mathrm{~cm}^{-1}$ band shift to $1174 \mathrm{~cm}^{-1}$ suggesting a strained solid is formed. It is observed that the solubility of barium sulfate in DMSO is higher than in water (presumably caused by a strong $\mathrm{Ba}^{2+\ldots} \mathrm{O}=\mathrm{S}\left(\mathrm{CH}_{3}\right)_{2}$ interaction), which explains the lower nucleation rates in DMSO compared to water at the same concentration. Intriguingly, there is a lack of nucleation observed (even at a relatively high supersaturation) when $\mathrm{Ba}^{2+}$ is solvated with DMSO supporting the hypothesis that de-solvation of the cation is the rate determining step in nucleation and is of higher activation energy in DMSO than in water
\end{abstract}

\section{Introduction}

The role of solvent is well established in crystallization (for example Mirmehrabi et al. ${ }^{1}$ ) and the use of anti-solvents is one of the most common crystallization methods in the pharmaceutical industry. ${ }^{2,3} \mathrm{~A}$ key feature of using anti-solvents is that they increase nucleation rates due to a higher driving force (caused by the lower solubility in the anti-solvent). However, the role of solvent other than its role in creating driving force is only little studied. To date, other than driving force, the solvent has only been investigated for its impact on morphology ${ }^{4-6}$ and occasionally on polymorph formation. ${ }^{7}$ Recently, some studies on calcium sulfate hydrates and the formation pathway to gypsum have raised some interesting questions about the role of water as solvent in many systems. ${ }^{8-}$ ${ }^{10}$ Thus, it is imperative that a more fundamental understanding of the solvent's role is undertaken if crystallization processes are to be predicted accurately.

In water, when salts are dissolved, an equilibrium will be established between solvated ion pairs and solvated ions. The interactions occurring are ion-ion and ion-solvent interactions and depending on the relative strengths of these interactions can lead to larger 'clusters'. One of the inorganic minerals proven to show a clustering mechanism in water has been the calcium carbonate system. ${ }^{11-13}$ In the case of calcium carbonate, water is critical in stabilizing both the liquid like clusters called

Curtin Institute for Functional Molecules and Interfaces, School of Molecular and Life Sciences, Curtin University.

† Electronic Supplementary Information (ESI) available: [Further SEM and TEM images as detailed in text, TGA and DLS information]. See DOI: 10.1039/x0xx00000x
DOLLOPs and the amorphous calcium carbonate (ACC) that forms in the initial stages.

In the case of barium sulfate crystallization, the 'two step mechanism' is hypothesised to occur. ${ }^{14-17}$ This states that initially, a highly disordered solid is formed and over time this becomes the crystalline particle via some mechanism, as predicted by Ostwald's rule of stages. Thus, the name 'two step' is used because crystallization occurs via two distinct steps formation of the disordered solid and then transformation to the crystalline solid. In addition, depending on the system, either removal of solvent or re-organisation to form the crystalline structure may be rate determining. If prenucleation cluster stability is related to dielectric constant reducing the dielectric constant of the solvent may be one way of increasing the nucleation rate. Thus, altering the solvent could be a means to promote the formation of the initial phase (amorphous or otherwise).

It is commonly found that as the dielectric constant of the solvent reduces the solubility of the salt reduces. ${ }^{18,19}$ As already mentioned, the stability of any pre-nucleation clusters might be expected to decrease as the dielectric constant decreases and as such nucleation rate increases even at the same supersaturation. ${ }^{19}$ Thus, the role of the solvent should be key to nucleation. ${ }^{20}$ However, the solvent can also play a part in growth and can impact on the morphology by changing the surface free energies in different solvents. Finally, different solvents may impact the way impurities interact with the crystallization processes. Impurities that are benign in water may be potent inhibitors in non-aqueous solvents.

In addition, the growth of inorganic particles need not be 'classical', i.e. through ion integration into the lattice at kink sites. Various non-classical mechanisms have been proposed from oriented attachment $(\mathrm{OA})^{21}$ to mesocrystals. ${ }^{22}$ In fact, systems where aggregation is the dominant growth mechanism 
have been known for some time (e.g. gibbsite grown under Bayer conditions, ${ }^{23}$ but their mechanistic underpinning is still not fully understood. Relatively recent literature has shown that water and the strength of the water interactions to the surface may be a driver in OA.$^{24,25}$

The studies on calcium sulfate dihydrate have shown that, in water, a hydrated amorphous calcium sulfate forms first and this transform to gypsum via the hemihydrate. ${ }^{8-10}$ While the amorphous calcium sulfate is not always observed in nonaqueous systems, the formation of the hemihydrate is and in some non-aqueous solvents does not transform to the dihydrate. The question is, what mechanisms are similar or different in the different solvents or for different mineral systems?

This manuscript, therefore, is investigating the role of dielectric constant on nucleation and growth of barium sulfate using DMSO as the solvent. The goal here is to understand how the dielectric constant impacts the pathways to nucleation and subsequent growth (classical or non-classical) for charged species and how this is altered as water is introduced into the system. Table 1 gives some important physical properties of the solvents discussed in this work.

Table 1. Physical Properties of water and $\mathrm{DMSO}^{26}$

\begin{tabular}{lcc}
\hline \multicolumn{1}{c}{ Physical Property } & Water & DMSO \\
\hline Dielectric Constant & 75.8 & 48.9 \\
Dipole moment $(\mathrm{D})$ & 1.85 & 3.96 \\
Viscosity $(\mathrm{cP})$ at $20^{\circ} \mathrm{C}$ & 0.890 & 1.991 \\
Refractive index $\left(20^{\circ} \mathrm{C}\right)$ & 1.3330 & 1.479 \\
Density $(\mathrm{g} / \mathrm{cm} 3)$ & 0.9971 & 1.0955 \\
Boiling point $\left({ }^{\circ} \mathrm{C}\right)$ & 99.98 & 189 \\
Surface tension $(\mathrm{mN} / \mathrm{m})$ & 72.86 & 43.54 \\
\hline
\end{tabular}

\section{Materials and Methods}

'Water free' DMSO was prepared by allowing neat DMSO (Merck, 99.9\% pure) to be kept in the presence of pre-dried and activated Molecular sieve type 5A for 24 hours. This was then decanted into $40-50 \mathrm{~mL}$ aliquots with $5-10 \mathrm{~g}$ pre-dried and activated Molecular sieve type $5 \mathrm{~A}$ for a further 24 hours prior to use. All solutions were kept in their container with the molecular sieve, wrapped with parafilm and kept in a desiccator with dry silica gel before using. Once opened what solvent was not used for the experiment was decanted into a fresh container with pre-dried and activated Molecular sieve type 5A. Ultra Violet spectroscopy was used to confirm the water content (see section below). After drying and removal of water, silica was removed by contacting the dry DMSO $(50 \mathrm{~mL})$ with barium sulfate solids $(2 \mathrm{~g})$ and this sample was also stored in the desiccator. All experiments were, however, conducted under normal laboratory ambient conditions with no special precautions to avoid moisture absorption from the air. Experiments with barium nitrate dissolved in 'dry' DMSO (see dynamic light scattering section) did not show any appreciable changes over time and so show that the dry DMSO resulted in water contents low enough to not impact our data. Moreover, these results show that absorption of moisture from the atmosphere was not significant enough to alter results.

\section{Water content measured by UV-vis}

In order to determine the water content of the DMSO after drying with the Molecular sieve type 5A (activated by heating for $\geq 24$ hours at $250^{\circ} \mathrm{C}$ ), the UV-vis spectrum of the sample was obtained and then further spectra were recorded as known amounts of water were added.

\section{Dynamic Light Scattering (DLS)}

Impact on nucleation rate was assessed via DLS. DLS of barite in DMSO and in water was undertaken by two methods:

a) by dissolving anhydrous barium nitrate $\left(56 \mathrm{mM} \mathrm{Ba}^{2+}\right.$ ) distributed into $20 \mathrm{~mL}$ vials at initial concentrations of 3.2, 6.4, 9.6 , and $12.8 \mathrm{mM}$ with equimolar quantities of concentrated $\mathrm{H}_{2} \mathrm{SO}_{4}$ dissolved in DMSO. Water was added to form $0,5,10$ v/v\% samples, making up to volume with DMSO. The solutions were shaken vigorously and allowed to sit for at least 24 hours for the crystals to form. The sample was then sonicated and analysed by the Malvern NanozS in a plastic cuvette to determine the derived counts and the particle size distribution. The calculated water content for this method is $0.2 \pm 1 \mathrm{v} / \mathrm{v} \%$.

b) by taking neat DMSO $(20 \mathrm{~mL}$, pre-dried) or ultrapure water $(20 \mathrm{~mL}$ ) and adding barium nitrate (anhydrous, varying volumes, $0.1 \mathrm{~mol} / \mathrm{L}$ in water) to a Teflon beaker and magnetically stirring at $200 \mathrm{rpm}$. Crystallization was commenced by adding equimolar quantities of $\mathrm{H}_{2} \mathrm{SO}_{4}(0.0184 \mathrm{~mol} / \mathrm{L}$ in water or DMSO). The water content of this sample when performed in DSMO was 3-4 ( $\pm 1 \%) v / v \%$ for all concentrations. The crystallization beaker was sampled at regular intervals by taking a $3 \mathrm{~mL}$ aliquot and analysed by the Malvern NanozS in a plastic cuvette to determine the derived counts and the particle size distribution. The $3 \mathrm{~mL}$ was returned to the beaker after analysis. Furthermore, tests were also conducted whereby both the barium nitrate and the sulfuric acid were dissolved in dry DMSO in order to ascertain whether there were any impacts of water on the nucleation behaviour.

The impact of silica on nucleation behaviour was determined by dissolving $\mathrm{SiO}_{2}$ (Ajax, 98\% pure) and $\mathrm{Ba}\left(\mathrm{NO}_{3}\right)_{2}$ into pre-dried DMSO. The silica level was increased to $20 \mathrm{mg} / \mathrm{L}$ (as measured by Inductively Coupled Plasma, ICP). The remaining process was kept identical to method (b). In addition, the pre-dried DMSO was left to sit with barium sulfate solids (AR grade, Sigma Aldrich) to adsorb $\mathrm{SiO}_{2}$ (and remove $\mathrm{SiO}_{2}$ from solution) before using in the nucleation experiments by method (b). This was confirmed to remove $\mathrm{SiO}_{2}$ (by ICP) and allowed us to ascertain the impact of variable $\mathrm{SiO}_{2}$ content.

\section{Solubility in DMSO}

The solubility of barium sulfate in DMSO was investigated to compare solubility products in the different solvents and to be able to determine relative supersaturation. Barium sulfate (Analytical reagent grade from Aldrich) was equilibrated with DMSO (having prior removed any $\mathrm{SiO}_{2}$ via adsorption) for $>1$ week at room temperature $\left(21-22^{\circ} \mathrm{C}\right)$. 
Inductively Coupled Plasma - Optical emission spectrometry (ICP-OES) method: Barium sulfate $(1 \mathrm{~g})$ was equilibrated in DMSO $(15 \mathrm{~mL})$ in a closed glass vial for several weeks in the laboratory at room temperature $\left(21-22^{\circ} \mathrm{C}\right)$. After this period, an aliquot was removed $(2 \mathrm{~mL})$. The supernatant DMSO was filtered through a $0.2 \mu \mathrm{m}$ PTFE membrane and evaporated to dryness. The solids were re-dissolved into nitric acid (68\%) and analysed by ICP-OES. This was then used to determine the barium ion concentration and the solubility product (assuming a 1:1 solid).

Microwave Plasma-Atomic Emission (MPAE) method: The filtered sample as described above was evaporated to dryness and reconstituted in $\mathrm{HNO}_{3}$. This method used known standards to determine the concentration of barium ion in the solvent. Three standards were prepared $(1,5,10 \mathrm{ppm})$ in $10 \% \mathrm{HNO}_{3}$, with each measured in triplicate. The pump speed was $15 \mathrm{rpm}$, sample uptake time was $20 \mathrm{~s}$ and the stabilisation time was $10 \mathrm{~s}$. Absorbance at both 455.5 and $614.2 \mathrm{~nm}$ was measured and the most linear correlation was kept. The correlation coefficient for Absorbance at $455.4 \mathrm{~nm}$ was 0.99876 while at $614.2 \mathrm{~nm}$ was found to be 0.99921 . This gave a value of barium ion dissolved in DMSO of $3.23 \pm 0.13 \mathrm{ppm}$ at room temperature (which was measured to be $21^{\circ} \mathrm{C}$ ). Conversion to molarity and assuming $1: 1$ relationship between barium and sulfate ions in solution gives $0.0235 \mathrm{mmol} \mathrm{Ba}^{2+}$ in solution.

\section{Transmission Electron Microscopy (TEM) and Scanning Electron Microscopy (SEM)}

The sample in DMSO was centrifuged, the bulk of DMSO decanted and subsequently re-dispersed in ethanol. The ethanol slurry was then used to prepare a TEM grid with the particles. The samples were viewed on a FEI Talos F200X FEGTEM with Super-X EDS system (Manufacturer: Thermo Fisher Scientific, Czech Republic).

For the samples in $100 \%$ water, the samples were allowed to crystallize onto a glass cover slide, which was collected, gently rinsed and placed on a carbon coated SEM stub. Excess fluid was removed with a tissue and the stub sputtered with $\mathrm{Pd}$. The sample was then viewed on a Zeiss Evo 40XVP SEM (Manufacturer: Zeiss, Germany) or Neon 40EsB Dual-Beam FESEM (Manufacturer: Zeiss, Germany). For samples in DMSO, the DMSO was filtered off and the residual solids were washed with ethanol before drying under vacuum.

\section{Results and Discussion}

\section{Solubility in DMSO}

The ICP results for barium in DMSO showed variability and so the alternative MPAE method was also used to determine the solubility. The results for the solubility product in each of the solvents are listed in Table 1 . According to these results, the solubility of $\mathrm{BaSO}_{4}$ in DMSO is 5-8 times more soluble than in water. In this manuscript the solubility as determined from the MPAE method was considered more reliable and was used for calculations. However, more accurate determinations are recommended in future.

\begin{tabular}{ccc}
\hline Table 2. $\mathrm{K}_{\mathrm{sp}}$ values for $\mathrm{BaSO}_{4}$ in $100 \%$ solvent & & \\
\hline Temperature $\left({ }^{\circ} \mathrm{C}\right)$ & Water* $^{*}$ & DMSO \\
21 & $1.0 \times 10^{-10}$ & $5.5-7.7 \times 10^{-10}$ \\
\hline${ }^{*}$ from PHREEQC ${ }^{27}$ & & \\
\hline
\end{tabular}

In addition, the ICP data showed that although $\sim 9$ ppm of Si was present in the DMSO after removal of water, this level dropped to $0.5 \mathrm{ppm}$ after contact with barium sulfate solids. This suggests that any silica present was adsorbing onto the barium sulfate particles. This effect was used to prepare low silica DMSO.

\section{Water content of dried DMSO}

Figure 1 shows the spectra obtained after adjusting the baselines to the same value for all spectra. The absorbance at $260 \mathrm{~nm}$ was found to be linear for water percentages less than $30 \%$ and this was used to calculate the water content of the DMSO after drying as $0 \pm 1 \%$.

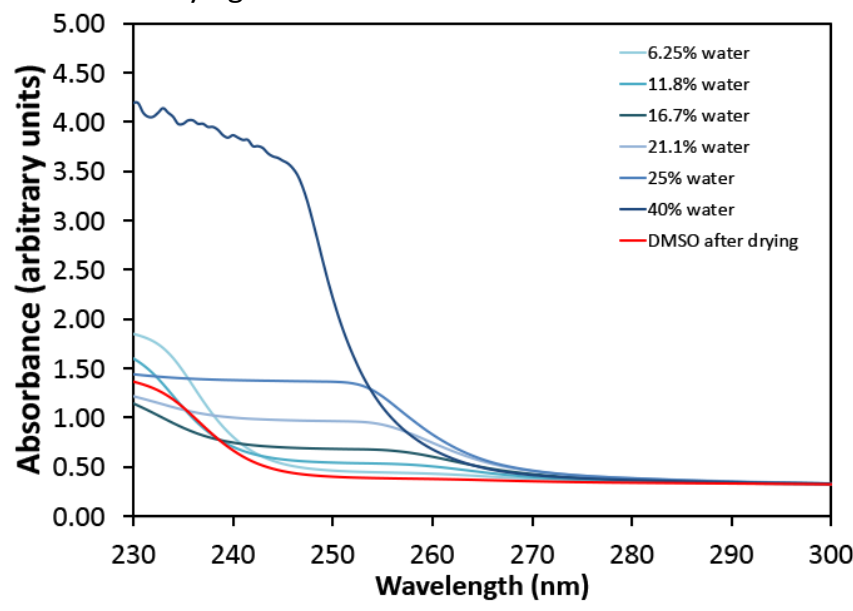

Figure 1. UV-vis spectrum of DMSO after drying and with known amounts of added water

\section{Morphology}

\section{$0.5 \mathrm{mM}$ initial concentration}

In water the barite particles are similar to that previously reported ${ }^{28,29}$ despite the $\mathrm{pH}$ being significantly lower than previous studies $\left(\mathrm{pH}=2.96\right.$ based on $\left[\mathrm{H}^{+}\right]$added). ${ }^{14,19,30}$ Essentially, pillow shaped particles (see supplementary section for more images, SFig. 1) bound by (001) faces and rounded (hk0) faces can be observed (Figure 2a). Protrusions are also observed on the particles resulting in a rough surface and there appear to be low density regions, three of which are marked with circles.

In DMSO and $0.5 \mathrm{mM}$ initial concentration, the particles were so few as to be not adequately imaged using SEM. SEM images of particles formed in dry DMSO were possible at higher concentration and one such image is shown in Figure $2 b$ for the $2 \mathrm{mM}$ case. The SEM shows that spherical aggregates formed in 


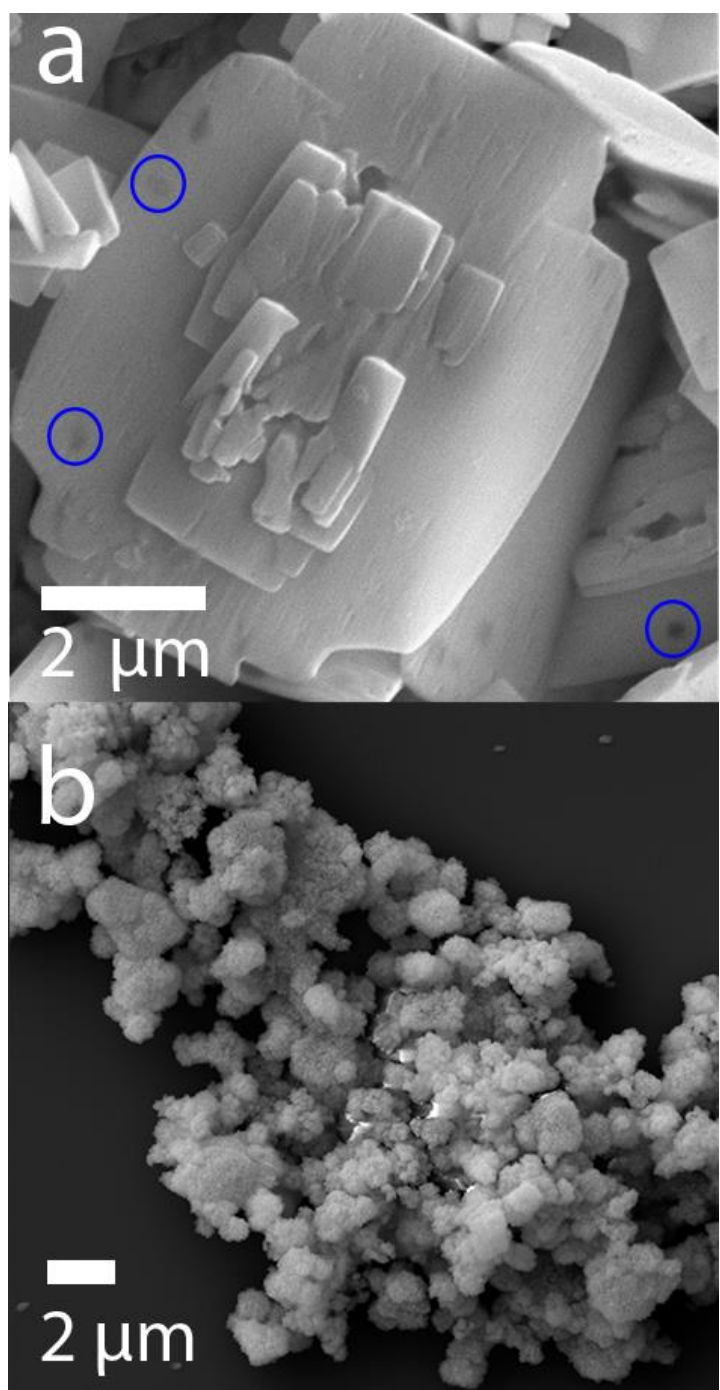

Figure 2. SEM images of barium sulfate formed in (a) water at $0.5 \mathrm{mM}$ $\mathrm{Ba}^{2+}$ initial concentration and (b) DMSO at $2 \mathrm{mM} \mathrm{Ba}^{2+}$ initial concentration

the micron range (Figure $2 b$ ) but appear to be made up of smaller particles.

Further analysis of the particles formed in DSMO was undertaken using TEM. The particles in dry DMSO at $0.5 \mathrm{mM}$ initial concentration, as viewed by TEM, appear to have two general morphologies. One morphology is shown in Figure $3 a$ and is rectangular-like but appears to be made up of rodlike particles. A close up of the particle (Figure 4a) shows it is generally crystalline in nature and has varied contrast. The crystallinity was confirmed through the FFT and the zone can be indexed as the $\langle 100\rangle$ zone (inset of Figure $3 a^{31}$ ). For the particle in Figure 3a this means that the long dimension in the particle is perpendicular to this zone, namely $<0 \mathrm{k} 1>$ or $\langle$ h10 $\rangle$. Interestingly the morphology seen in Figure $3 a$ has similarities to that observed in the presence of silicate previously seen ${ }^{32}$ (see supplementary information SFig. 2). The morphology in Figure $3 b$ is more diamond-like and reminiscent of the dendritic or diamond form of barium sulfate (see SFig. 3 in the supplementary section). The FFT

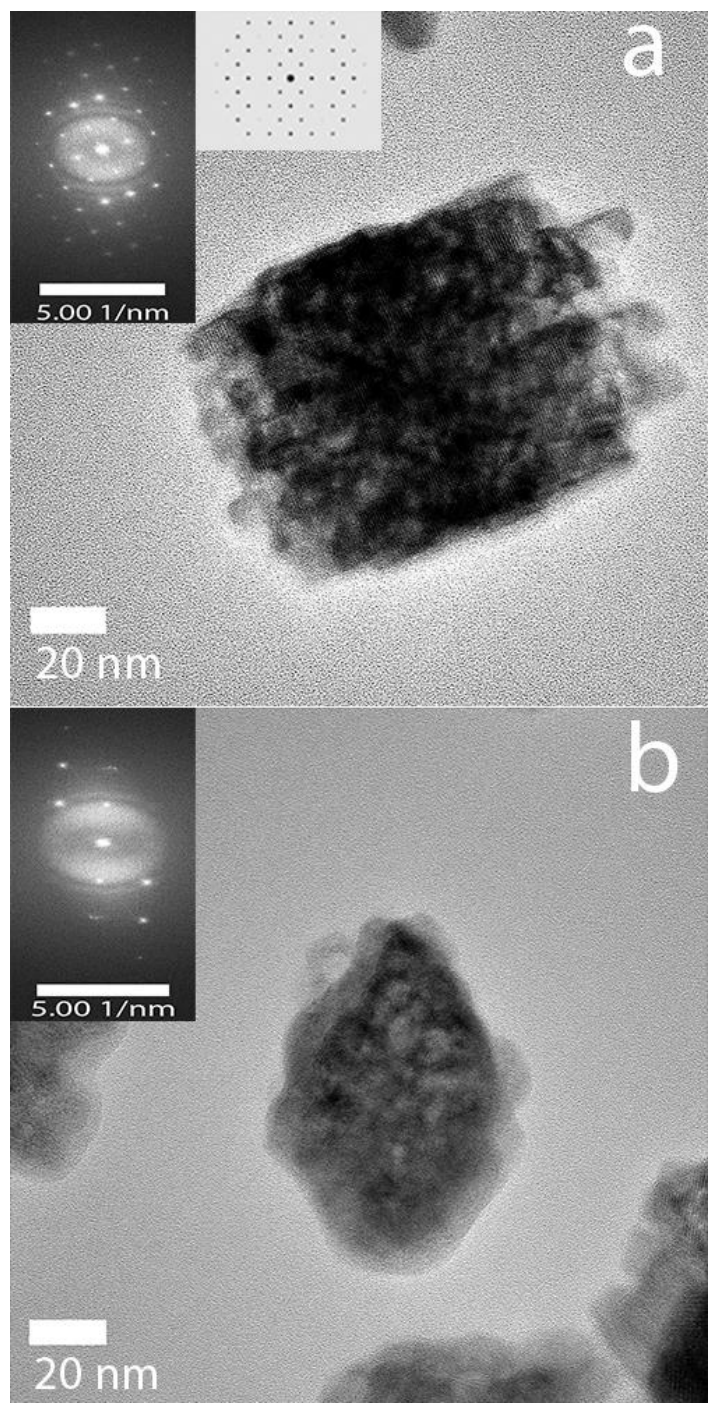

Figure 3. TEM images of the two morphologies of barium sulfate identified in DMSO and their respective FFT patterns as insets. The second inset in Figure $3 a$ is the calculated SAED for the barium sulfate $\langle 100\rangle$ zone from SingleCrystal ${ }^{\circ}$

of the particle in Figure $3 \mathrm{~b}$ is slightly distorted and cannot be assigned conclusively.

The variation in contrast could be due to:

- the solvent being occluded within the structure,

- the transformation from an amorphous solid to a crystalline one (which has a greater density).

- Lattice mismatch and/or nanoparticle coalescence creating voids.

Previous literature on barium sulfate crystallisation in water hypothesized that smaller particles come together to form larger structures $^{15,17}$. 


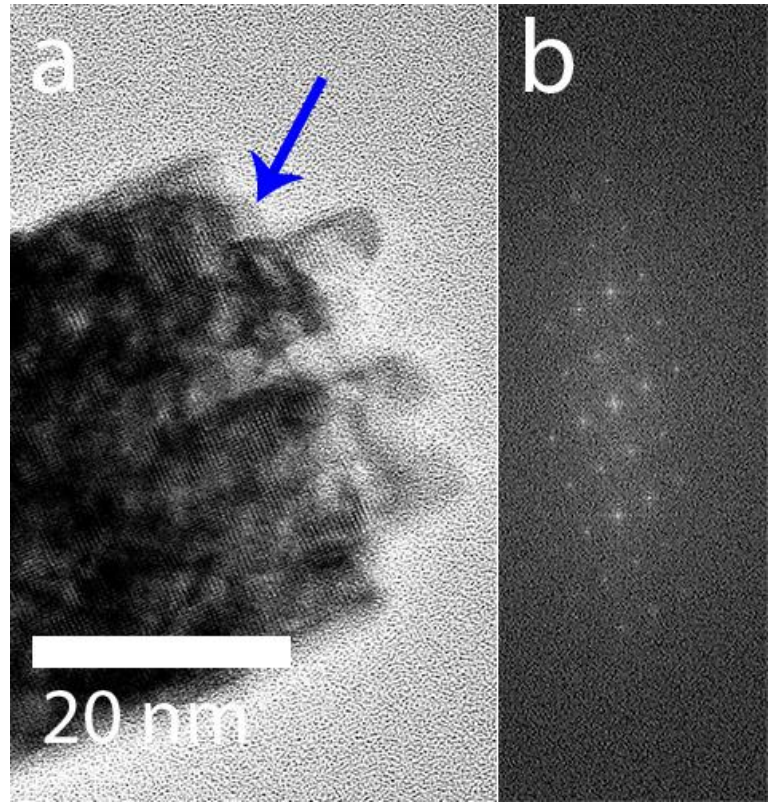

Figure 4. (a) Close up of the particles shown in Figure 3a showing crystalline extremity, and (b) Fourier transform of central region shown in (4a)

It is interesting to note that particles formed in either solvent show contrast or density variations, thus whatever mechanism is occurring it is common to both solvents.

Elemental mapping of particles (Figure $5 a, b, c$ ) confirmed barite but also showed particles are surrounded by oxygen and silicon. The lack of $S$ with $O$ suggests that the $O$ is not associated with residual DMSO but, as the EDX spectrum confirmed in Figure 5c, indicates $\mathrm{SiO}_{2}$. Contact with barium sulfate solids reduced $\mathrm{Si}$ content according to the ICP data and is supported by EDX (see supplementary information SFig. 4). Initially, it was hypothesized that $\mathrm{SiO}_{2}$ was intimately involved in the formation of these particles. However, no significant difference in the morphology (see supplementary information SFig. 5) was observed. Entrapped DMSO solvent in the structure was assessed by looking at the mass fraction of $\mathrm{Ba} / \mathrm{S}$ in the EDX results. A mass ratio of 4.29 (or an atomic ratio of $1: 1$ ) would suggest pure $\mathrm{BaSO}_{4}$. However, the mass ratio was found to be 5.2 suggesting that more barium is present than $S$ in the solid. The atomic fractions for this sample showed Ba 5.44, S 4.08, Si 0.73 .

TGA (see supplementary section SFig. 6) on this sample found that above $180{ }^{\circ} \mathrm{C}$ (approximately the boiling point of DMSO) at most 2.5 wt\% of DMSO was adsorbed on the solids. Below 180 ${ }^{\circ} \mathrm{C}$ any weight loss was assumed to be due to residual DMSO not adsorbed DMSO. If we assume spherical $20 \mathrm{~nm}$ radius particles this is $400 \mathrm{~nm}^{2} /$ molecule DMSO - not a high density).

\section{2-5 mM initial concentration}

At 2-5 mM initial concentration for barium nitrate and sulfuric acid in DMSO, the supersaturation ratio (defined as $c / c_{o}$ for initial concentration $\mathrm{c}$ and equilibrium concentration $\mathrm{c}_{0}$ ) was calculated to be $72-85$ for $2 \mathrm{mM}, 180-200$ for $5 \mathrm{mM}$ (depending on solubility product used) and in the case of water (at $0.5 \mathrm{mM}$ ) it is expected to be 55 . In the SEM images (see Figure 6), it
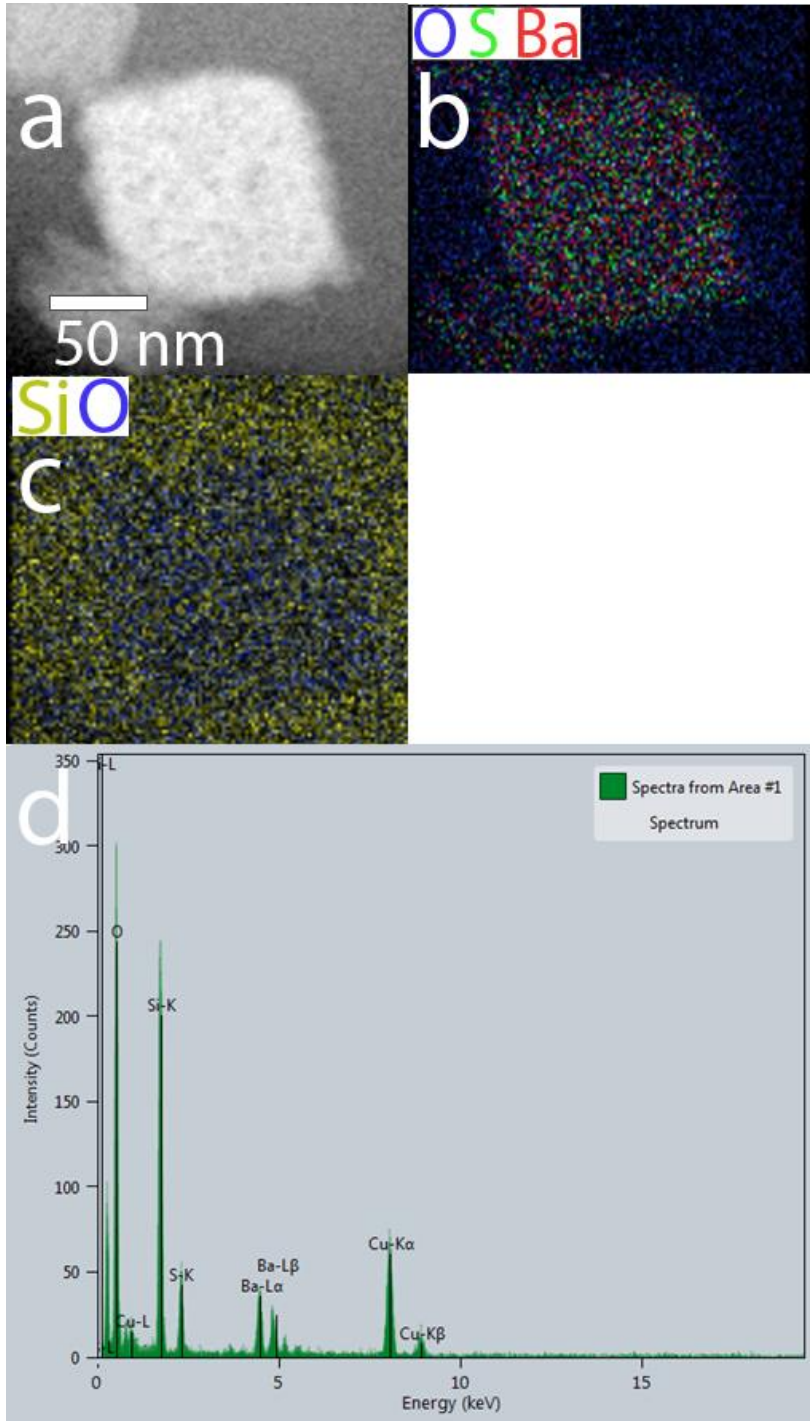

Figure 5. (a) HAADF image (b) Ba, S, O EDX elemental map, (c) Si, O EDX elemental map and (d) EDX spectrum of sample shown in (a)

appeared that the solids consisted of roughly spherical 'particles'. However, these solids could also be made up of smaller primary particles. Thus, this sample was also observed in the TEM and showed that the roughly spherical aggregates were indeed made up of much smaller particles (Figure 7 and supplementary information SFig. 7). Whether the aggregates are coalesced could not be ascertained conclusively at this point. EDX mapping was also able to confirm the solids were barium sulfate (see supplementary information SFig. 8 ). 


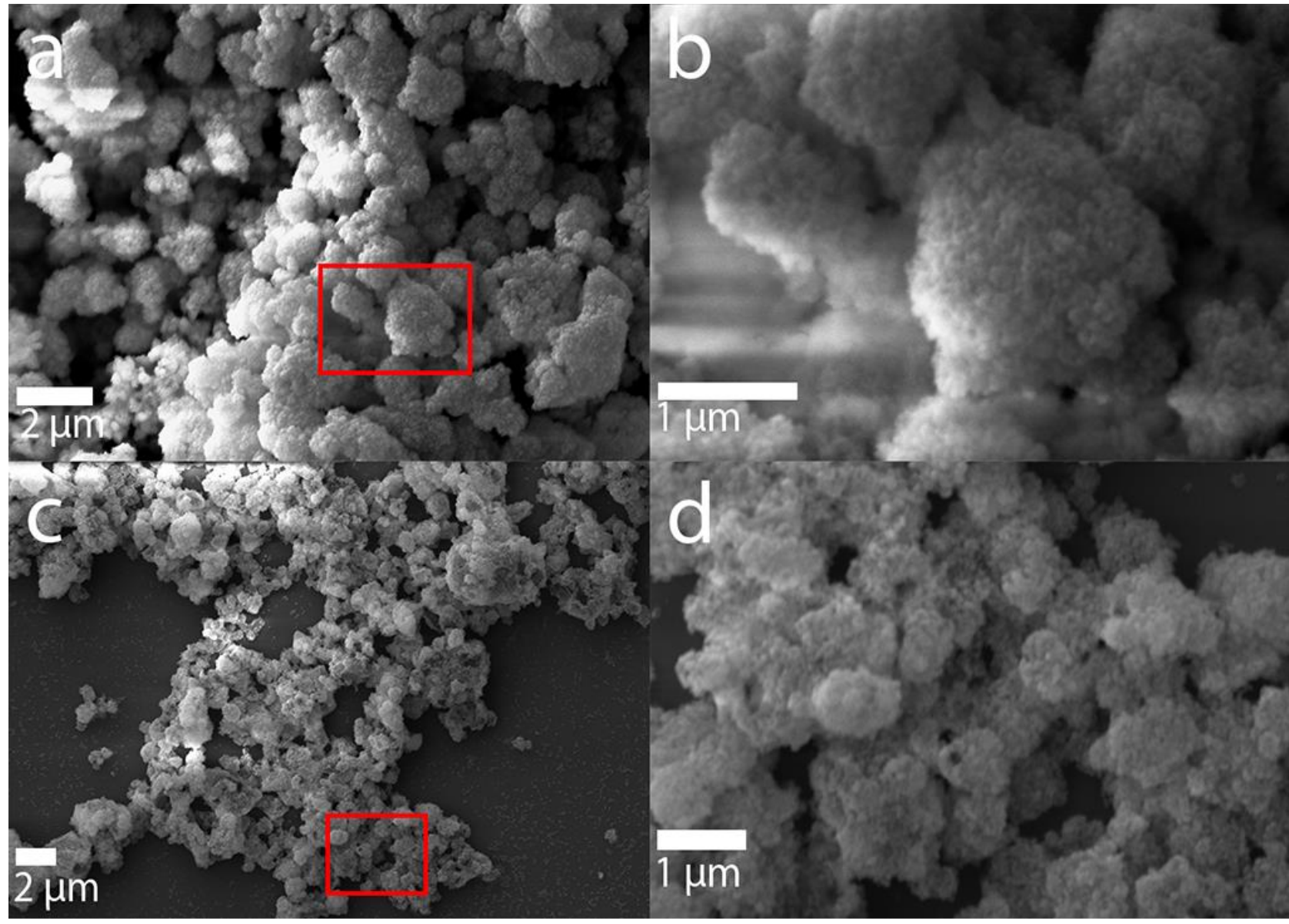

Figure 6. SEM images of barium sulfate particles formed at (a), (b) $2 \mathrm{mM}$, and (c), (d) $5 \mathrm{mM} \mathrm{Ba}^{2+}, \mathrm{H}_{2} \mathrm{SO}_{4}$ in DMSO. Sections in red box have been enlarged.

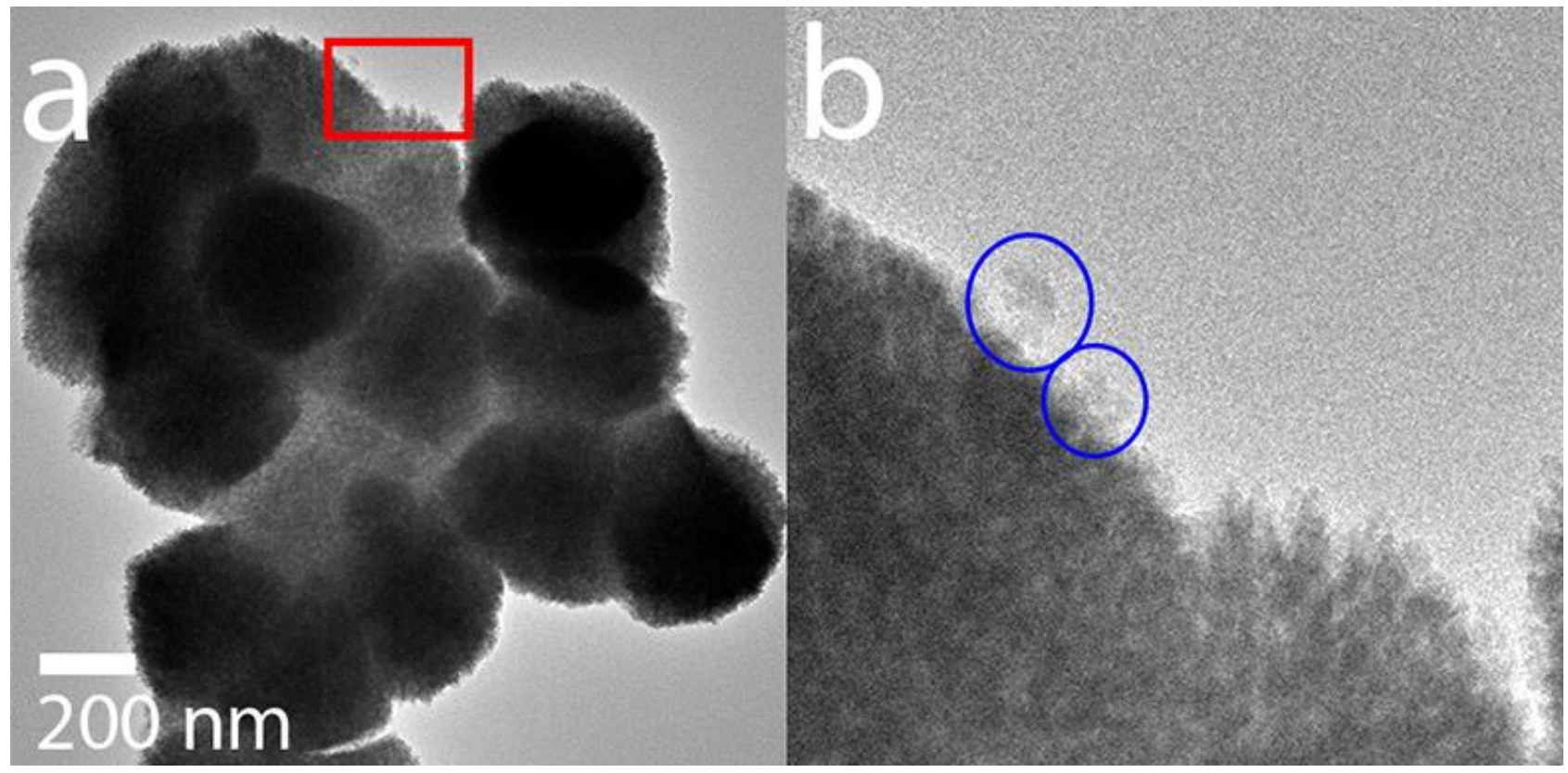

Figure 7. TEM image of barium sulfate particles formed at (a) $5 \mathrm{mM} \mathrm{Ba}^{2+}, \mathrm{H}_{2} \mathrm{SO}_{4}$ in DMSO and (b) expanded region in red box with small particles highlighted by blue circles 


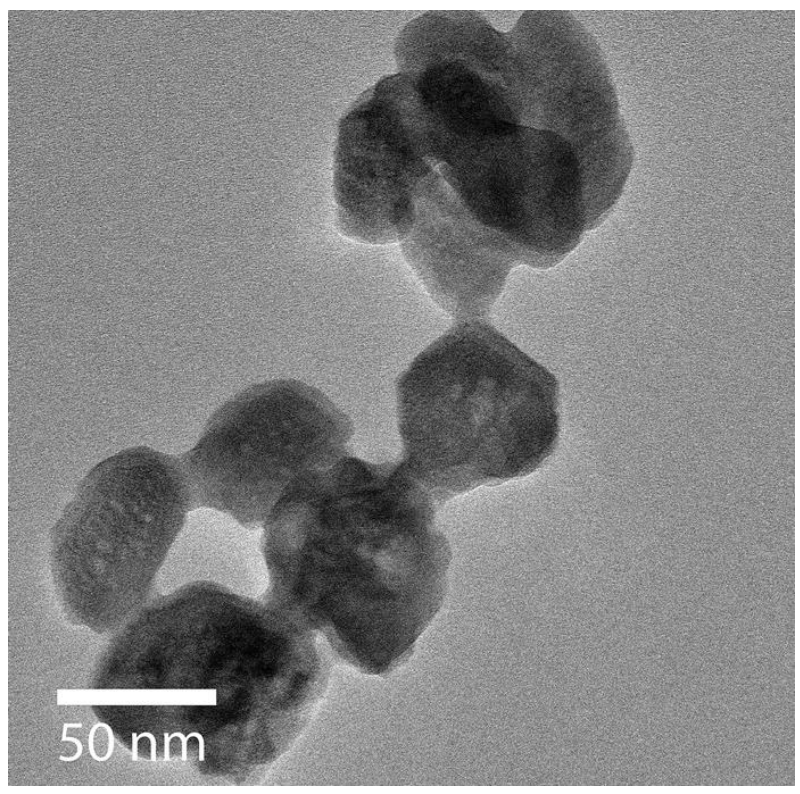

Figure 8. TEM image of $0.5 \mathrm{mM} \mathrm{BaSO} 4$ particles formed in DMSO with added water ( $5 \mathrm{wt} \%$ ) post particle formation

\section{Impact of water}

To the $0.5 \mathrm{mM}$ sample in DMSO, water (5 wt\%) was added to see what changes occurred to the particles. In DMSO, the particles were in the 50-100 $\mathrm{nm}$ range and aggregated/agglomerated particles were at most two particles large. The addition of water appeared to promote the coalescence of several individual particles into aggregates (see Figure 8). This was also monitored by DLS and showed that after the addition of water the particle number increased from approximately 1100 to $3600 \mathrm{kcps}$ (suggesting more nucleation occurred) and the average size increased to a value fluctuating around $190 \mathrm{~nm}$, suggesting these aggregates were fused together.

The regions in the neck section joining one particle to another appear amorphous in character (see supplementary information SFig. 9) although this is difficult to determine conclusively. Regardless, the behaviour on addition of water is easily understood by the difference in barite solubility in the solvents; as solubility is lower in water, supersaturation increases, resulting in further precipitation and cementation is promoted by the additional precipitation.

If water is present from the start a slightly different impact is observed (Figure 9). At a water level of $60 \mathrm{v} / \mathrm{v} \%$ at $0.5 \mathrm{mM}$, the particles formed showed significant aggregation and particles have considerable low electron density in regions. The particles are still crystalline but the shape of the individual particles is more reminiscent of barite particles formed in water despite being much smaller.

Increasing the water content further to $80 \mathrm{v} / \mathrm{v} \%$ (Figure 10) showed aggregation is again important and the overall aggregate shape now is somewhat similar to particles found in water.

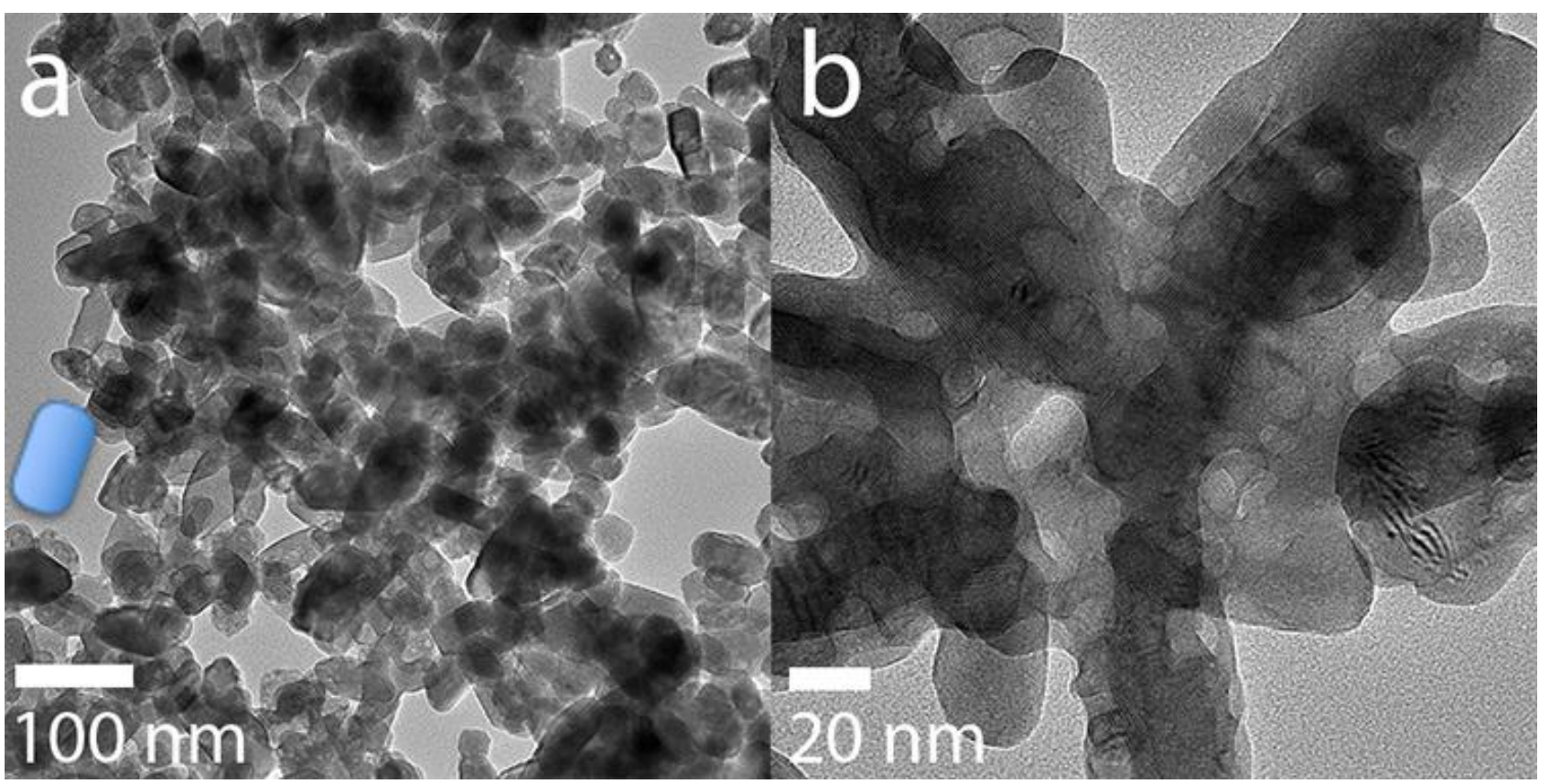

Figure 9. (a) TEM images of barium sulfate particles formed in the presence of $60 \mathrm{v} / \mathrm{v} \%$ water at $0.5 \mathrm{mM} \mathrm{Ba}^{2+}, \mathrm{H}_{2} \mathrm{SO}_{4}$. Blue rectangle shows the general shape of the individual particles within this aggregate. (b) high magnification image of the same sample 


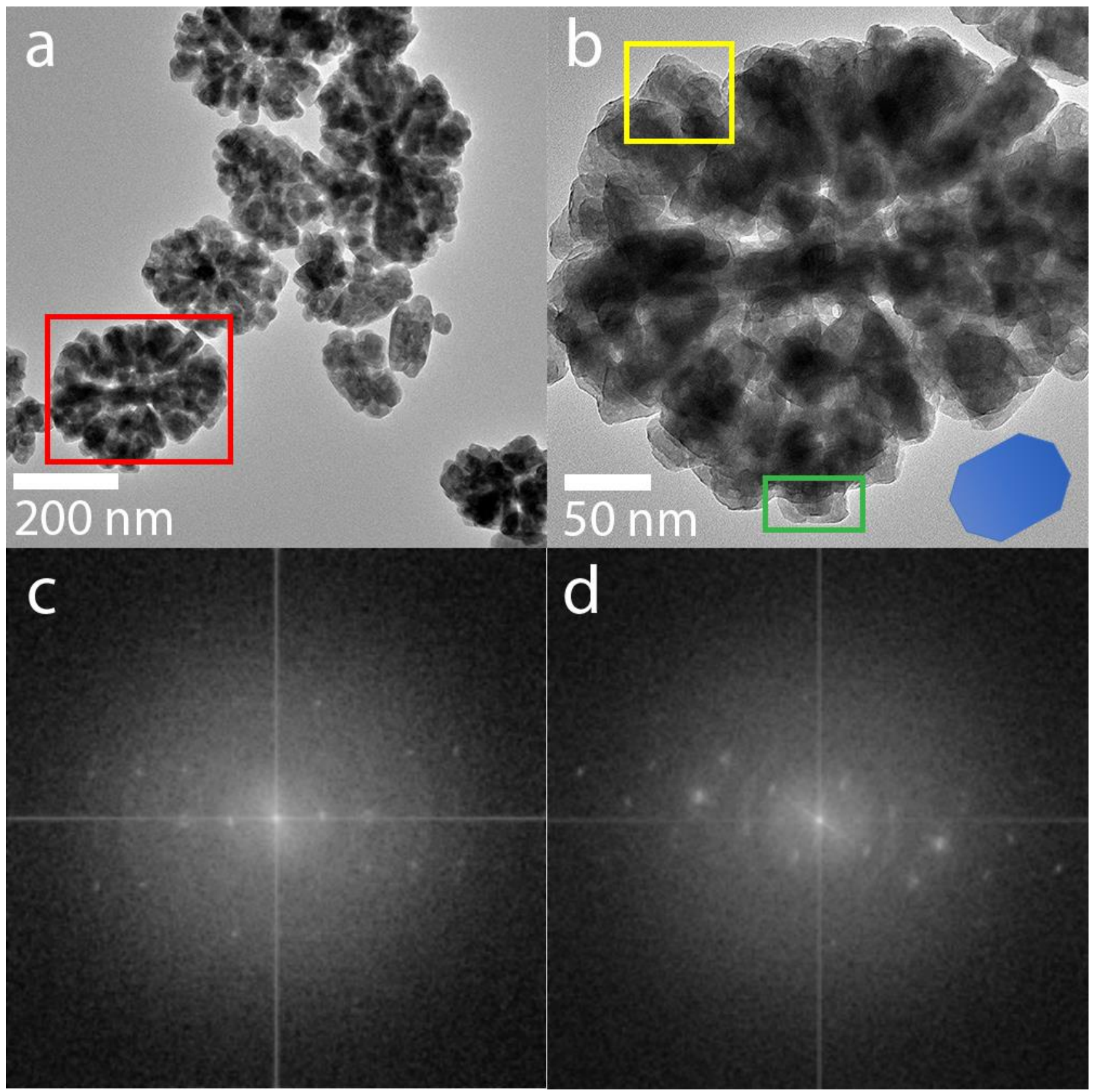

Figure 10. (a) TEM images of barium sulfate particles formed in the presence of $80 \mathrm{v} / \mathrm{v} \%$ water at $0.5 \mathrm{mM} \mathrm{Ba}^{2+}, \mathrm{H}_{2} \mathrm{SO}_{4}$ and (b) expanded aggregate in red box. Blue octagon insert shows the general shape of the aggregates. (c), (d) FFT of regions shown in yellow and green boxes

These particles are approaching the surface tension and dielectric of water so should approach a similar morphology/size as water content increases. However, even at $80 \mathrm{v} / \mathrm{v} \%$ water, the size of the particles is $100-300 \mathrm{~nm}$, much less than the micron sized particles in water.

\section{Kinetics and Nucleation (DLS)}

The number of particles formed can be measured using DLS. The expected behaviour is that the number of particles begin low (homogenous solution) then grows quickly due to nucleation. Particle numbers should remain stable once nucleation is complete but can decrease due to aggregation and/or sedimentation of particles. The results at an initial concentration of $0.5 \mathrm{mM}$ are presented in Figure 11. Note, that since the solubility product is lower for $\mathrm{BaSO}_{4}$ in water that the supersaturation is higher in this solvent. 


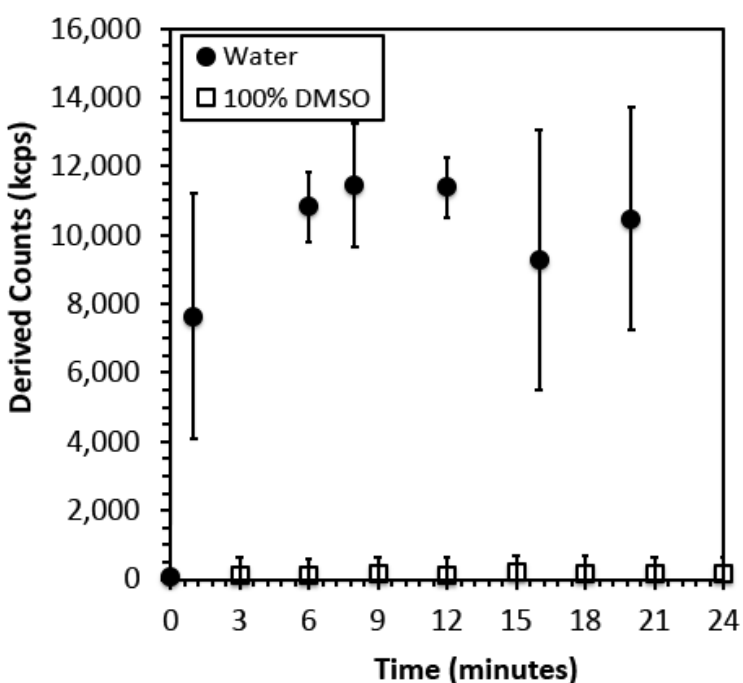

Figure 11. Derived counts per second versus time for pure water and dried DMSO (0.5 $\mathrm{mM} \mathrm{Ba}^{2+}$ and $\left.\mathrm{SO}_{4}{ }^{2-}\right)$

It is clear that in water at an $\mathrm{S}\left(\mathrm{c} / \mathrm{c}_{\mathrm{o}}\right)=55$ nucleation essentially ceases after 5-10 minutes, plateauing out at particle numbers of between 10000-14000 kilocounts per second overall. If the supersaturation is lower in DMSO the nucleation rate should also be lower. This is supported by the results where it takes up to 5 hours to reach the maximum in particle numbers recorded ( $2000 \mathrm{kcps}$ ).

While previous studies have shown that silicate does not influence nucleation $32,34,35$ of barite this may not be true in other solvents. Additional $\mathrm{SiO}_{2}$ was dissolved in DMSO to investigate the impact on nucleation rate. When $\mathrm{SiO}_{2}$ was present in the DMSO as well as barium and sulfate ions, the nucleation rate was effectively hindered to the point where little to no nucleation occurred (see Figure 12a). The particle numbers increased from $75 \mathrm{kcps}$ to $195 \mathrm{kcps}$ over the analysis period. Thus, silica is a nucleation inhibitor in DMSO. Of even greater interest however, was the impact on nucleation rate when the silica was essentially removed by adsorption. As can be seen in Figure 12b, nucleation did not improve. Thus, while high levels of silica can impact on nucleation - at the levels DMSO has after drying, no impact on nucleation is observed. In addition, the particles formed at low Si levels were of a similar morphology to those observed previously (see supplementary information SFig. 4, 5).
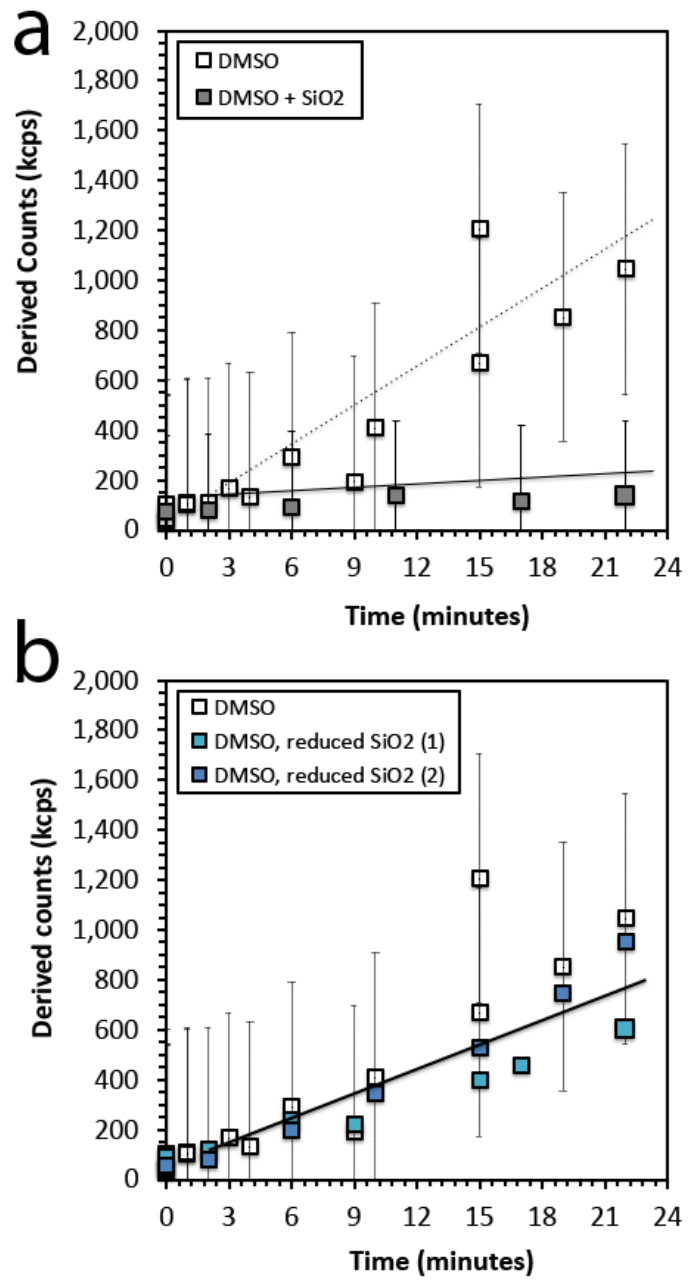

Figure 12. Derived counts versus time for different Si levels present during barium sulfate crystallisation in DMSO. a) Dried DMSO has $\sim 9 \mathrm{ppm}$ silica present, $20 \mathrm{ppm}$ was present in the $\mathrm{DMSO}+\mathrm{SiO}_{2}$ sample and b) $\sim 0.6$ ppm was present in the reduced $\mathrm{SiO}_{2}$ samples.

The DMSO also appears to limit the size that the particles attain. Figure 13 shows the evolution of the particle size with time. The particles do not grow appreciably over the course of the experiment. Looking at the $24 \mathrm{hr}$ sizes with respect to water content, it is clear that the presence of water leads to larger crystals. In addition, at a fixed water content, larger particles are formed with higher $\mathrm{Ba}^{2+}$ and $\mathrm{SO}_{4}{ }^{2-}$ concentrations.

When the concentration of the barium nitrate and sulfuric acid is increased in DMSO to 2-5 mM, a different behaviour is observed. Firstly, if the barium nitrate is prepared in DMSO then there is little to no nucleation observed (see supplementary information SFig. 10). This is irrespective of whether some water is present with the sulfuric acid or not (up to $3 \pm 1 \mathrm{v} / \mathrm{v} \%$ ). However, when the barium nitrate is prepared in water and the final water content is $2 \mathrm{v} / \mathrm{v} \%$ the nucleation rate is increased as would be expected (see Figure 14). These experiments were conducted on low $\mathrm{Si}$ solutions thus the issue is not due to $\mathrm{SiO}_{2}$ contamination. Clearly, the nucleation rate is similar for $5 \mathrm{mM}$ in DMSO and $0.55 \mathrm{mM}$ in water. Despite the higher calculated supersaturation at $5 \mathrm{mM} \mathrm{Ba}^{2+}, \mathrm{SO}_{4}{ }^{2-}$ in DMSO the nucleation 
rate is only just comparable to that in water. Also, the primary particle sizes or the aggregates are still much smaller in DMSO than water (as Figure 13b shows the primary particle size is 50 $70 \mathrm{~nm}$ at $5 \mathrm{mM}$ initial $\left[\mathrm{Ba}^{2+}\right]$ while aggregates are also smaller, Figure 6).
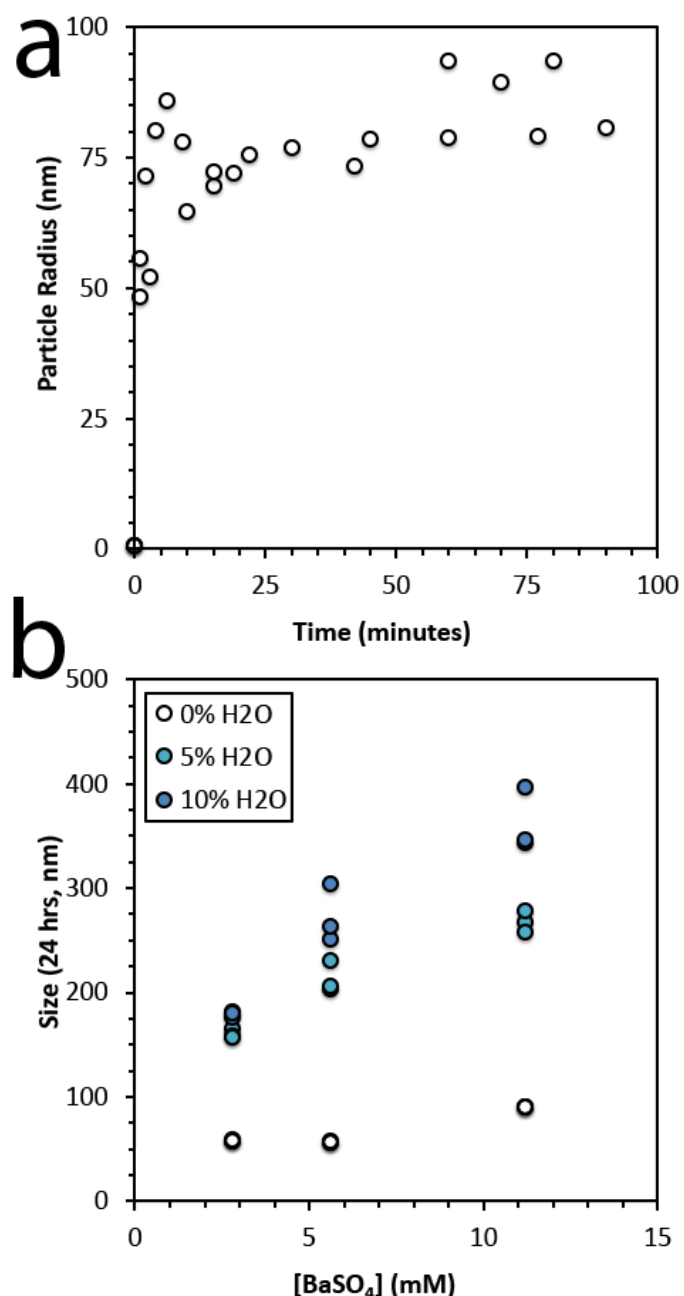

Figure 13. (a) Barium sulfate particle size measured by DLS in dried DMSO at $0.55 \mathrm{mM} \mathrm{Ba}^{2+}$ and $\mathrm{SO}_{4}{ }^{2-}$ (b) barium sulfate particle size measured after 24 hours by DLS in DMSO and water mixtures and at different concentrations

\section{Infrared}

Monitoring of the crystallization via infrared spectroscopy was made difficult by the fact that DMSO has bands within the same range as sulfate. Despite this, infrared was attempted as per ref ${ }^{14}$ whereby the crystallization was initiated by addition of sulfuric acid. The subtracted spectra are shown in Figure 15. The solids all display crystalline spectra from very early on but the value of the expected $1200 \mathrm{~cm}^{-1}$ is observed much lower at $1174 \mathrm{~cm}^{-1}$ suggesting a strained or lower symmetry solid is formed. ${ }^{36,37}$ This is consistent with the TEM results that show a highly crystalline solid but significant lattice mismatching (see supplementary information SFig. 11). It is impossible to tell whether the $1075 \mathrm{~cm}^{-1}$ is altered as this band is too heavily influenced by the large DMSO peak in this region. In addition, the spectra show an increasing band intensity for some bands that are related to DMSO. This could indicate that DMSO is adsorbed on the surface even if, as found for the TEM preparation, it is easily displaced by ethanol.

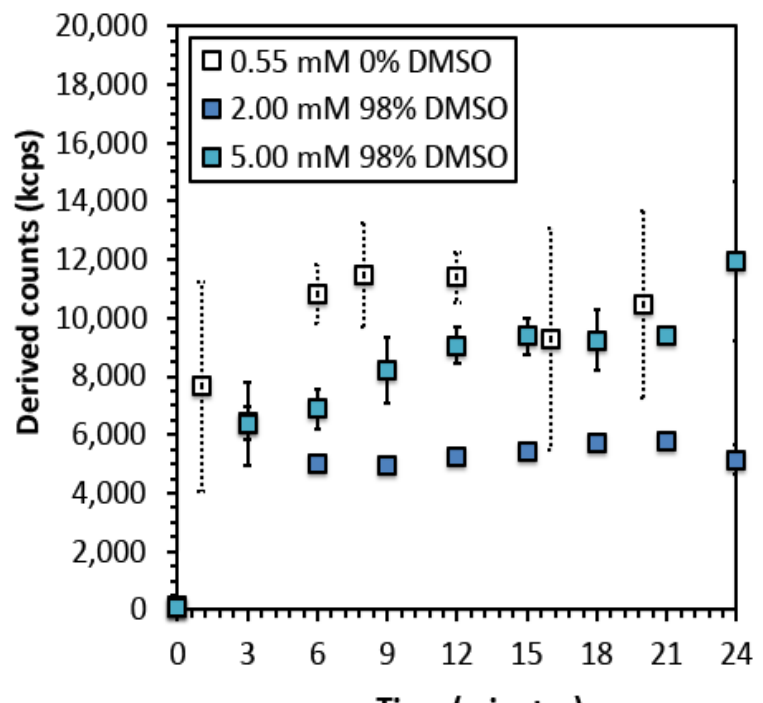

Figure 14. Derived counts versus time for barium sulfate formed in water and DMSO to match supersaturation.

\section{Discussion}

When comparing the different solvents, the factors that can strongly influence the nucleation rate are the supersaturation and the change in the interfacial free energy, $\gamma$, that appears as the third power in the exponential term. In classical nucleation theory, according to the Gibbs-Thomson equation, interfacial free energy impacts on the nucleation rate $J: 20$

$J=\mathrm{A} \cdot \exp \left(-\Delta G_{c}\right)$ with $\Delta G_{c}=16 \pi \gamma^{3} V^{2} 0 / 3 k^{3}{ }_{\mathrm{B}} T^{3} \ln \left(S^{*}\right)^{2}$

Eqn 1.

where $\gamma$ is the interfacial free energy, $V_{0}$ is the volume of a unit cell, and $S^{*}$ is the supersaturation. According to Eqn 1 increasing the interfacial free energy should decrease the nucleation rate while increasing supersaturation should increase nucleation rate. Given the solubility of barium sulfate in the two solvents, at the same concentration, of say $0.5 \mathrm{mM}$, the nucleation rate should follow water $(\mathrm{S}=55)>$ DMSO $(\mathrm{S}=18-21)$. This is indeed what is observed.

It is also worth mentioning that once particles are nucleated they will grow until the ion concentration reaches the solubility limit in a batch system. Thus, if more nuclei are formed the final size is expected to be smaller than if fewer particles are nucleated.

The particles formed in DMSO did not show any evidence of two-step nucleation (whereby an amorphous solid transforms to a crystalline one). In addition, amorphous solids were not readily observed in the infrared whereby all bands corresponded to crystalline barium sulfate from a relatively early time. Combined with the TEM information where lattice 
mismatching is observed, it suggests that in DMSO the transition from amorphous to crystalline has a very low activation energy barrier making it even shorter lived than in water if present at all. If amorphous material was depositing on pre-existing material and crystallising slowly on contact this should not result in lattice mismatch. Thus, this does not appear to be occurring here. This lack of amorphous material is also seen in the case of calcium sulfate hemihydrate formation. ${ }^{8-10}$ As previously mentioned, the variation of contrast in the particles could be due to three possible mechanisms. While the EDX data suggests that the silicate could incorporate slightly into the solid, the important point is that it does not suggest extensive DMSO incorporation (no excess $S$ observed), thus solvent entrapment into the structure is minimal. This also means that trapped solvent can be excluded as a source of the porosity seen in the TEM images. As no amorphous to crystalline transition could be observed, this leaves the coalescence of smaller particles into a larger structure as the only possible mechanism.

The supersaturation of barium sulfate in water is high enough that it homogenously nucleates. However, this may not be the case in DMSO. The overall counts for the DMSO system is lower than in water (Figure 11). This could mean that at $\mathrm{S}=18-21$ in DMSO the system is in the metastable zone or just at a very low nucleation rate. In addition, the increased viscosity of DSMO may lower the collision frequency/diffusivity and result in an even lower nucleation rate. ${ }^{38}$ The relative difference for the solvents in terms of viscosity is significant, DMSO viscosity is $\sim 2$ times higher than that for water. Viscosity is often neglected but would be critical in impacting nucleation through collision rates (and impingement of ions/clusters). Thus, as viscosity increases the collision rate wil decrease and so too will the nucleation rate.

A reduction in nuclei size is to be expected when supersaturation increases. So it is unusual that the particles are smaller when DMSO is the solvent rather than water (at $0.5 \mathrm{mM}$ ). But the surface tension of DMSO is less than water (see Table 1) so if it is assumed that the surface tension is related to the interfacial tension, an increase in nucleation rate is expected for the DMSO solvent when supersaturation is matched along with a size decrease (in batch systems). If barium sulfate is assumed to have a critical nucleus size of $0.24 \mathrm{~nm}$ this would lead to an expected critical size in DMSO at the same supersaturation of $0.09 \mathrm{~nm} .{ }^{33}$

DMSO has a higher dipole moment than water and this would suggest that the $\mathrm{Ba}^{2+\ldots} \mathrm{O}=\mathrm{S}\left(\mathrm{CH}_{3}\right)_{2}$ interaction may be stabilized in this solvent. It is known that the de-solvation of the barium ion in water is the rate determining step in aqueous crystallisation of barite. ${ }^{20} \mathrm{It}$ is also possible that de-solvation of the barium ion is also critical in DMSO - leading to even lower nucleation rates if removal of DMSO requires more energy. In fact, the absence of any significant nucleation (even at $S=180-200$ ) when no aqueous $\mathrm{Ba}^{2+}$ was present (barium nitrate in DMSO case, see supplmentary information SFig. 10) suggests that the activation energy for removing the solvation shell of DMSO is high enough to limit nucleation. This is supported by work on calcium-DMSO

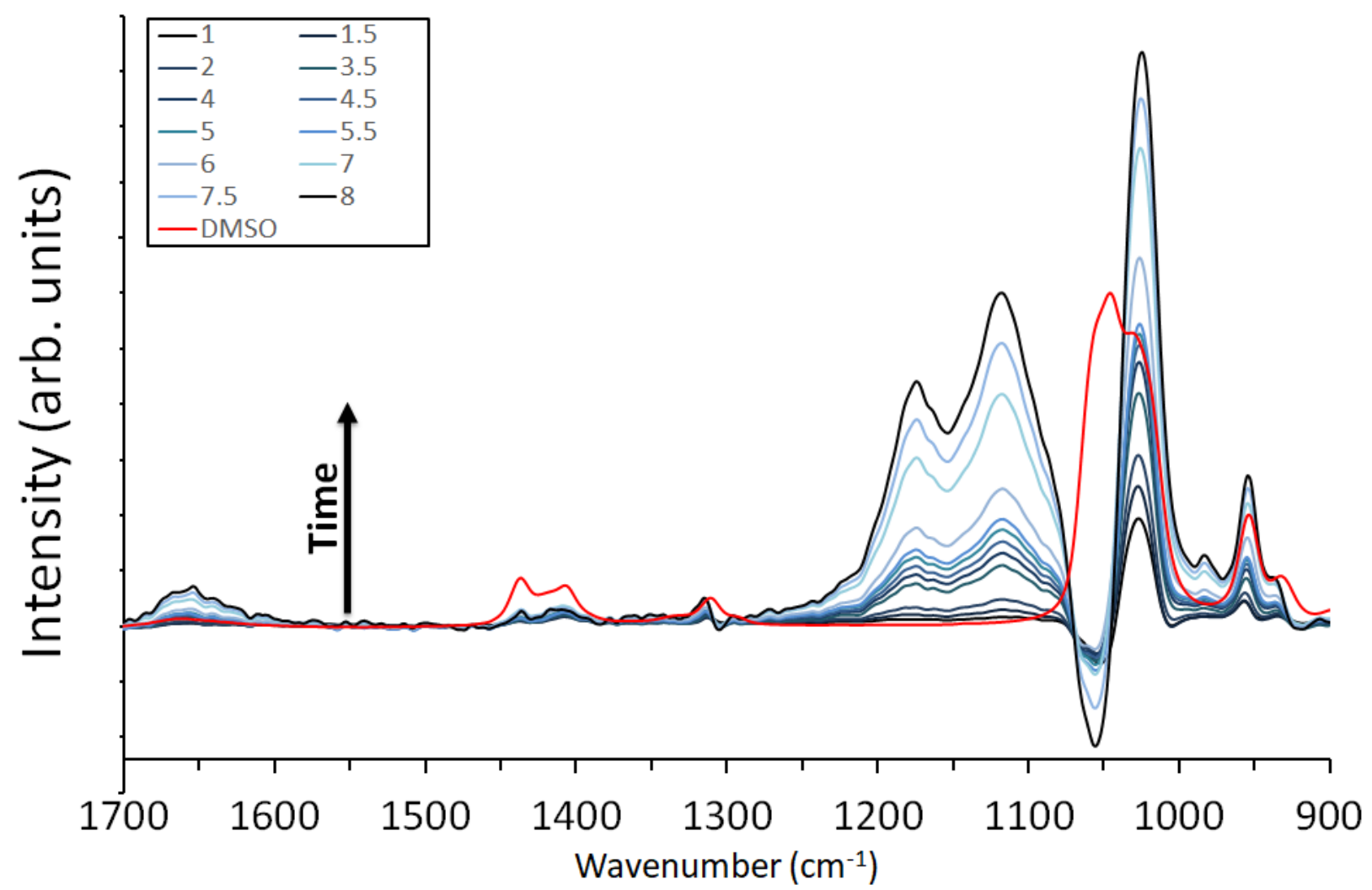

Figure 15. FTIR spectrum of barium sulfate particles formed versus time in the 1700 to $900 \mathrm{~cm}^{-1}$ region. Numbers in legend refer to minutes elapsed. Pure DMSO is highlighted in red. 
complexes that show that removal of the DMSO requires more energy than removal of water by almost $100 \%{ }^{39}$

All the results combined show that both in the presence and absence of water, the growth mechanism is through addition of nanoparticles not through a classical ion-by-ion addition. Thus, growth in both systems is similar in that respect. It is just that in water the process is highly oriented while in DMSO it is less so with misalignments occurring, leading to strained particles. Generally, in both systems, larger particles are formed via fusion of nanoparticles along matching lattice directions (as observed by Ruiz-Agudo et al. ${ }^{15,17}$ ). The aggregates formed in DMSO are roughly spherical; suggestive of a more random process. Despite this, small regions of the particles show both crystallinity and alignment but also show misaligned fusion particularly when little water is present (see supplementary section SFig. 11, 12). Thus, the process is not completely random. Also, in DMSO, there is less fusion into larger particles occurring as the smaller final size at $80 \%$ water content shows. The energy required to produce an aggregate does appear to be lower than that of coalescence or re-orientation of lattice misaligned particles. Thus, the hypothesis is that the dielectric constant of the solvent plays an important role in whether OA mechanisms are likely to dominate (see schematic in Figure 16). This is supported by the recent work of de Yoreo showing water layers are important in OA. ${ }^{24,25}$

In the absence of water it appears that the aggregates seen in the TEM or SEM do not fuse or coalesce on a $24 \mathrm{hr}$ time frame. This can be seen from the SEM images that show spherical aggregates (Figure $2 \mathrm{~b}$ ) but the DLS at $24 \mathrm{hrs}$ (Figure 14) shows particle sizes of $\sim 50 \mathrm{~nm}$. In water, coalesce appears to be instantaneous.

The impact of solvent on size is of interest since it is normally understood that when a batch system nucleates less, the particles can grow to larger sizes. This is not occurring in this case. It is interesting to note that it only takes the presence of $\sim 5 \mathrm{v} / \mathrm{v} \%$ of water to increase the size of particles by $\geq 2$ fold. In DMSO there remain unanswered questions:
- Why do the primary particles not grow any further in size? (see Figure 3, supplementary information SFig. 11)

- Why is aggregation preferred over growth? (See Figure 6 \& 7)

The particles formed in DMSO are significantly smaller than those formed in water, even when aggregate size is considered (cf Figure 2a and 6) and supersaturation is better matched. Nanoparticles of $\mathrm{BaSO}_{4}$ and $\mathrm{CaSO}_{4} \cdot 2 \mathrm{H}_{2} \mathrm{O}$ were also observed by Comet et al. ${ }^{40}$, when using acetone as the anti-solvent. Counterintuitively as the concentration of barium and sulfate increases so too does the size, despite an increase in nucleation rate. This effect is best explained by particle aggregation. As to why the primary particles formed in DMSO are smaller this could be due to the DMSO acting as an inhibitor. It may adsorb onto the surface and hinder the solids from coalescing with each other. As shown in the infrared and TGA data, there does appear to be some evidence of adsorption onto the surface of the barium sulfate particles. Having said this, any adsorbed DMSO appears to be displaced by ethanol during the TEM preparation, such that little to no additional $\mathrm{S}$ is observed in the EDX analysis. Thus, it is unclear at this point whether this mechanism is sufficient to explain the smaller sizes.

Most interesting is that in the absence of sufficient water around the barium ion nucleation was absent. In high levels of DMSO, even when water was surrounding the barium ion and supersaturation was higher than that in water, the nucleation rates were not higher than in water as would be expected. This may be in large part due to the viscosity of the solvent but suggests that nucleation kinetics are more sensitive to their environment than previously thought.
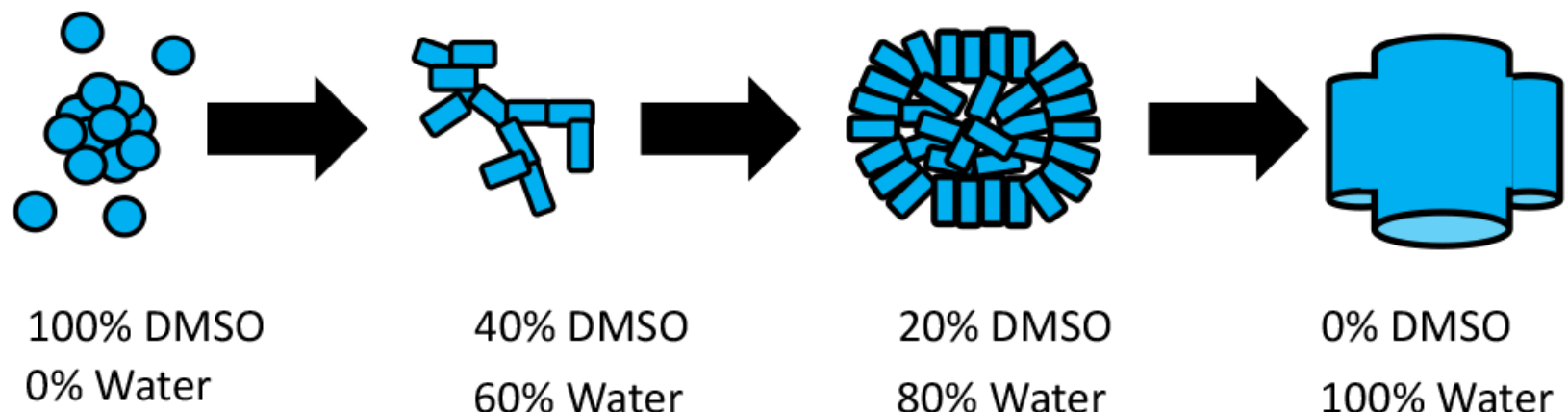

$40 \%$ DMSO

$20 \%$ DMSO

0\% DMSO

$60 \%$ Water

$80 \%$ Water

\section{$100 \%$ Water}

\section{Dielectric Constant}

Figure 16. Schematic of particle size/shape relationship with dielectric constant magnitude 


\section{Conclusion}

To summarise, the use of DMSO as the solvent in the crystallization of barite results in small primary particles. These primary nanoparticles tend to aggregate rather than grow further by an ion addition mechanism. The solubility of barite in DMSO is actually higher than in water, leading to low nucleation numbers when ion concentrations (but not supersaturation) are matched to those in water. It is surmised that this is due to the

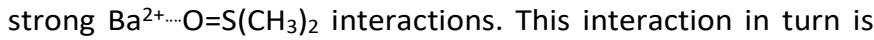
due to the large dipole moment in DMSO.

In water, aggregation is also evident, particularly when a mixture of DMSO and water is present. The aggregation process is through oriented attachment when water is dominant. In this case, there is evidence of fusion of particles when there is registry between the atomic positions. In DMSO, however, the aggregates are almost spherical and while there is lattice registry there is also cementation of non-perfectly-aligned particles (supplementary information SFig. 11). Thus, the dielectric constant of the solvent appears to determine the extent of OA possible.

The crystals in both systems were crystalline and no evidence of an amorphous precursor was observed when DMSO was the solvent, suggesting no such precursor pathway or there is a very fast transition to the crystalline state. Barium sulfate does not have a crystalline polymorph and so unlike the case with calcium sulfate the use of a non-aqueous solvent does not show stabilisation of the kinetic product. ${ }^{8-10}$ In addition, the FTIR results showed crystalline solids forming from a very early stage.

The main impact in decreasing the dielectric constant of the solvent appears to be:

- the influence on the solubility of the solute

- the fusion process into larger particles is less ordered as less polar interactions are present to drive reorientation processes and much slower than in water. Perhaps more interestingly,

- barium ion desolvation appears to be key, so much so that little nucleation is observed when barium is not added as an aqueous species.

\section{Conflict of interest}

There are no conflicts of interest to declare.

\section{Acknowledgements}

We would like to thank and acknowledge the John de Laeter Centre, Curtin University for access to the EM/XRD (ARC LE0775553). We would also like to acknowledge the Australian government for funding via the Australian postgraduate award and Curtin University for support of J. A. Saunders.

\section{References}

1

2

3

4

5

6

8

9
M. Mirmehrabi and S. Rohani, J. Pharm. Sci., 2005, 94, 1560-1576.

M. Maghsoodi, Adv. Pharm. Bull., 2015, 5, 13-18.

Z. Q. Yu, R. B. H. Tan and P. S. Chow, J. Cryst. Growth, 2005, 279, 477-488.

J. R. Bourne and R. J. Davey, J. Cryst. Growth, 1976, 36, 278-286.

N. Sanchez-Pastor, C. M. Pina, J. M. Astilleros, L. Fernandez-Diaz and A. Putnis, .

C. Stoica, P. Verwer, H. Meekes, P. J. C. M. van Hoof, F. M. Kaspersen and E. Vlieg, Cryst. Growth Des., 2004, 4, 765768.

J. Wang, C. Loose, J. Baxter, D. W. Cai, Y. L. Wang, J. Tom and J. Lepore, J. Cryst. Growth, 2005, 283, 469-478.

A. E. S. Van Driessche, L. G. Benning, J. D. RodriguezBlanco, M. Ossorio, P. Bots and J. M. García-Ruiz, Science (80-. )., 2012, 335, 69-72.

Y. W. Wang, Y. Y. Kim, H. K. Christenson and F. C. Meldrum, Chem. Commun., 2012, 48, 504-506.

U. Tritschler, M. Kellermeier, C. Debus, A. Kempter and H. Cölfen, CrystEngComm, 2015, 17, 3772-3776.

R. Demichelis, P. Raitieri, J. D. Gale, D. Quigley and D. Gebauer, Nat. Commun., 2011, 2, 1-8.

D. Gebauer and H. Cölfen, Nano Today, 2012, 6, 564-584.

D. Gebauer, A. Völkel and H. Cölfen, Science, 2008, 322, 1819-22.

F. Jones, Crystengcomm, , DOI:10.1039/c2ce25918d.

C. Ruiz-Agudo, E. Ruiz-Agudo, C. V Putnis and A. Putnis, Cryst. Growth Des., 2015, 15, 3724-3733.

Z. Dai, F. Zhang, A. T. Kan, G. Ruan, F. Yan, N. Bhandari, Z. Zhang, Y. Liu and A. Y.-T. Lu, Ind. Eng. Chem. Res., 2019, 58, 10864-10874.

C. Ruiz-Agudo, E. Ruiz-Agudo, A. Burgos-Cara, C. V Putnis, A. Ibanez-Velasco, C. Rodriguez-Navarro and A. Putnis, Crystengcomm, 2016, 18, 2830-2842.

W. G. Gorman and G. D. Hall, J. Pharm. Sci., 1964, 53, 1017-1020.

F. Jones, S. Piana and J. D. Gale, Cryst. Growth Des., 2008, 8, 817-822.

S. Piana, F. Jones and J. D. Gale, J. Am. Chem. Soc., 2006, 128, 13568-13574.

X. Xue, R. L. Penn, E. R. Leite, F. Huang and Z. Lin, CrystEngComm, 2014, 16, 1419-1429.

S. H. Yu, H. Cölfen and M. Antonietti, Chem. - A Eur. J., 2002, 8, 2937-2945.

I. Seyssiecq, S. Veesler, R. Boistelle and J. M. Lamèrant, Chem. Eng. Sci., 1998, 53, 2177-2185.

L. Liu, E. Nakouzi, M. L. Sushko, G. K. Schenter, C. J. Mundy, J. Chun and J. J. de Yoreo, Nat. Commun., 2020, 11, 10451056.

S. N. Kerisit and J. J. de Yoreo, J. Phys. Chem. C, 2020, 124, 5480-5488.

Gaylord Chemicals, Dimethyl Sulfoxide Physical Properties, Sildell, CA, 2019.

D. L. Parkhurst and C. A. Appelo, Users guide to PHREEQC 
(version 2) - a computer program for speciation, batch reaction, one dimensional transport, and inverse geochemical calculations, U.S. Geological Survey WaterResources Investigation Report, 1999.

T. Radomirovic, M. I. Ogden and F. Jones, J. Cryst. Growth, 2011, 321, 78-84.

29 F. Jones, A. Oliviera, G. M. Parkinson, A. L. Rohl, A. Stanley and T. Upson, J. Cryst. Growth, 2004, 270, 593-603.

30 C. Ruiz-Agudo, C. V Putnis, E. Ruiz-Agudo and A. Putnis, Chem. Geol., 2015, 391, 7-18.

31 F. Jones, W. R. Richmond and A. L. Rohl, J. Phys. Chem. B, 2006, 110, 7414-7424.

32 F. Jones, T. Radomirovic and M. I. Ogden, Cryst. Growth Des., 2012, 12, 3057-3065.

33 F. Jones, M. I. Ogden, G. M. Parkinson and A. L. Rohl, CrystEngComm, 2005, 7, 320-323. M. Boon and F. Jones, Cryst. Growth Des., 2016, 16, 46464657. R. T. Kügler, K. Beißert and M. Kind, Chem. Eng. Res. Des., 2016, 114, 30-38.

36 H. H. Adler and P. F. Kerr, Am. Mineral., 1965, 50, 132-147.

37 J. J. Wylde, G. C. Allen and I. R. Collins, Appl. Spectrosc., 2001, 55, 1155-1160.

38 H. Wang, Q. Lin, X. Dou, T. Yang and Y. Han, Crystals, 2017, 7, 357-367.

39 M. Jakl, M. Straka, J. Jaklová Dytrtová and J. Roithová, Int. J. Mass Spectrom., 2014, 360, 8-14.

40 M. Comet, G. Vidick, F. Schnell, Y. Suma, B. Baps and D. Spitzer, Angew. Chemie - Int. Ed., 2015, 54, 4458-4462. 